\title{
Miscible Blends Comprising Two Carbonyl-Containing Polymers. Poly(E-caprolactone) with Poly(benzyl methacrylate)
}

\author{
Tarun K. Mandal and Eamor M. Woo ${ }^{\dagger}$ \\ Department of Chemical Engineering, National Cheng Kung University, \\ Tainan, 701, Taiwan
}

(Received June 19, 1998)

\begin{abstract}
Differential scanning calorimetry, optical microscopy, and infrared spectroscopy were perfomed to characterize poly( $\varepsilon$-caprolactone) (PCL)/poly(benzyl methacrylate) (PBzMA) blend of full compositions. The binary polymer mixture of all compositions forms a true miscible blend with a homogeneous phase structure attributed to matched polarity. The thermal transition behavior suggests that the intermolecular interactions between the pairs are likely non-specific and only comparable with those among the homopolymer molecules. The FT-IR results infers that inter-molecular mixing in segmental scales does exist, although the segmental scales are not particularly fine and some minor homomolecular aggregation might be present. It is concluded that the blend phase behavior is miscibility with non-specific, mostly being of a matched polarity, intermolecular interactions. Upon heating, phase separation occurs via a binodal nucleation \& growth (NG) mechanism at above $240^{\circ} \mathrm{C}$. The phase separation phenomenon is attributed to a cause of thermodynamic lower critical solution temperature (LCST) behavior. Upon cooling at regularly reasonable rates, phase separation could be reversed back to original homogeneity of miscibility.

KEY WORDS Polycaprolactone / Poly(benzyl methacrylate) / Miscibility / Fourier Transform Infrared / Lower Critical Solution Temperature/
\end{abstract}

Poly( $\varepsilon$-caprolactone) (PCL) has been known to be miscible with many polymers. The miscibility of PCL with poly(vinyl chloride) (PVC) is one of the most well known. Following the initial demonstration of PCL/PVC miscibility by Koleske and Lundberg, ${ }^{1}$ many studies have been performed to investigate this interesting $\mathrm{PCL} / \mathrm{PVC}$ system. In addition, miscibility of PCL with other polymers have since extensively been reported. Although PCL is neither miscible with polystyrene nor with polyacrylonitrile, PCL turns out to be miscible with the copolymers of styrene and acrylonitrile (especially PSAN with $28 \mathrm{wt} \% \mathrm{AN}$ ), and McMaster reported lower critical solution temperature (LCST) behavior in this blend. ${ }^{2}$ PCL is miscible with some hydroxyl-containing polymers, among which poly(hydroxyl ether of bisphenol-A) (phenoxy) might be one of the most notable. Using dynamic mechanical analysis, Brode and Koleske ${ }^{3}$ reported miscibility with the $T_{\mathrm{g}}$ of $\mathrm{PCL} /$ phenoxy blend satisfying the Fox equation. By contrast, miscibility of PCL with carbonyl- or carbonate-containing polymers has not been common. Earlier, Paul et al. ${ }^{4}$ reported miscibility in the blend of PCL with bisphenol-A polycarbonate (PC), which might be one of the rare cases. Miscibility has not been reported for blends of PCL with any of acrylate polymers, although some chlorinated acrylate polymers may exhibit affinity and thus miscibility with PCL. For example, poly(chloromethyl methacrylate) and poly(2-chloroethyl methacrylate) have been shown to be miscible with PCL by Neo and Goh. ${ }^{5}$

Complete miscibility in blends comprising two homopolymers containing carbonyl groups has not been common. An earlier study indicated that blends of PC with poly(ethylene terephthalate) (PET) exhibit partial miscibility and phase separation occurs for PC/PET blend with PET contents greater than $50 \mathrm{wt} \%{ }^{4}$ Although miscibility in two homopolymers both with carbonyl groups is not common, copolymerization in one the constituents might alter the polarity and its affinity. For example, copolymerization of PET might lead to an adjusted polarity that may lead to miscibility in blends of the PET copolymer with PCL. Nishi, et al. ${ }^{6}$ have reported that miscibility occurs in the blend of PCL with poly(ethylene terephthalate-co-caprolactone). More lately, Cimimno et al. reported miscibility (of amorphous region) in blend of poly(D- $(-)-3$-hydroxybutyrate and poly(methyl methacrylate). ${ }^{7}$ An interesting but highly controversial example is given by the binary blend of bisphenol-A PC with poly(methyl methacrylate) (PMMA), whose phase behavior has been extensively investigated by many investigators. ${ }^{8,9}$ Some claimed that miscibility existed in PC/PMMA with an LCST phenomenon (located around $160-190^{\circ} \mathrm{C}$ depending on compositions). But more recent studies have disputed that thermodynamic miscibility exists in PC/PMMA, but concluded that temporary kinetic trapping of polymer molecules might lead to apparently single $T_{\mathrm{g}}$ behavior. ${ }^{10}$ Kinetic trapping into temporary homogeneity (by fast solution casting) in PC/PMMA has also been studied by Motowoka et al. ${ }^{11}$ using a small-angle neutron scattering (SANS) technique. A more recent study in this laboratory also concluded that partial miscibility with micro-heterogeneity domains is a better description of the true phase behavior for PC/PMMA. ${ }^{12}$

The objective of this study was to examine possibility of miscibility in blends of polyesters (carbonyl in main chains) with acrylate- or methacrylate-type polymers (pendant carbonyl). PCL was chosen as the model polyester, and its possible miscibility or interactions with polymethacrylate polymers was examined.

\footnotetext{
${ }^{\dagger}$ To whom correspondence should be addressed (Fax: +886-6-234-4496, Phone: +886-6-275-7575 Ext. 62670, e-mail: emwoo@mail.ncku.edu.tw).
} 


\section{EXPERIMENTAL}

\section{Materials and Sample Preparation}

PCL was supplied from Polyscience, Inc. (U.S.A.), with an approximate $M_{w}=35000-45000 \mathrm{~g} \mathrm{~mol}^{-1}$ with a literature $T_{\mathrm{g}}=-60^{\circ} \mathrm{C}$ and $T_{\mathrm{m}}=60^{\circ} \mathrm{C}$. Poly(benzyl methacrylate) (PBzMA) was purchased from Scientific Polymers Product, Inc. (SP ${ }^{2}$, U.S.A.), with an approximate $M_{w}=70000 \mathrm{~g} \mathrm{~mol}^{-1}$ (GPC), and a $T_{\mathrm{g}}$ of $54-60^{\circ} \mathrm{C}$. Note that the $T_{\mathrm{g}}$ 's of these two polymers differ widely by at least $100^{\circ} \mathrm{C}$. The as-received materials were free of additives and were used without further purification.

Several solvents were used for sample preparation. Tetrahydrofuran (THF), chloroform $\left(\mathrm{CH}_{3} \mathrm{Cl}\right)$, and dichloromethane $\left(\mathrm{CH}_{2} \mathrm{Cl}_{2}\right)$ were used for preparing most blend samples in this study. As the temperature of casting was expected to influence the result of blend morphology owing to kinetic process of solvent evaporation, the blend samples in this study were prepared by solvent-casting at a range of temperature. Specifically two different temperatures were used: 20 and $45^{\circ} \mathrm{C}$, respectively. The polymers were first weighed respectively and dissolved into the solvent with continuous stirring with a stirrer or magnet. Subsequently, the resulting polymer solution after complete dissolution was poured into an aluminum mold kept at one of the two temperatures $\left(25\right.$ or $\left.45^{\circ} \mathrm{C}\right)$. The solvent in the cast film samples was first vaporized under a hood at a controlled temperature, followed by final solvent removal in a vacuum oven for $48 \mathrm{~h}$ at $50-60^{\circ} \mathrm{C}$. The THF-cast films at either of the two temperatures $\left(45\right.$ and $\left.25^{\circ} \mathrm{C}\right)$ visually appeared to be transparent. On the other hand, the blend films cast from methylene chloride or chloroform solutions visually appeared cloudy. When observed using an optical microscope for preliminary examination, the blend samples cast with dichloromethane or chloroform (at ambient temperature or $40^{\circ} \mathrm{C}$ ) exhibited an apparently phase-separated structure, indicating solvent effects and necessity of selection of proper solvents. In this study, the PCL/PBzMA blend samples for subsequent analysis were primarily cast films prepared from THF blending and casting at $45^{\circ} \mathrm{C}$. It must be noted here that for this blend, samples from $\mathrm{THF}$ casting at $25^{\circ} \mathrm{C}$ also yielded same results.

\section{Apparatus}

Differential Scanning Calorimetry. The glass transition temperatures and other thermal transitions of neat polymers (PCL and PBzMA) and their blends were measured with a differential scanning calorimeter (PerkinElmer DSC-7) equipped with a mechanical intracooler and a computer for data acquisition/analysis. Sub-ambient runs at temperatures lower than $-50^{\circ} \mathrm{C}$ were cooled with liquid nitrogen tank and helium purge. All $T_{\mathrm{g}}$ measurements were made at a scan rate of $20^{\circ} \mathrm{C} \mathrm{min}^{-1}$, and the $T_{\mathrm{g}}$ values were taken as the onset of the transition (the change of the specific heat) in the DSC thermograms.

Optical Microscopy. A polarized-light microscope (Nikon Optiphot-2, POL) was used. The as-cast blends were spread as thin films on glass slides, dried properly in a temperature-controlled oven before they were ex- amined using the optical microscope. For comparison, samples for optical examination were prepared using the same solvents and casting temperature as those used in preparing the thermal analysis samples. Cloud point measurement of the blends was performed by placing the samples on a microscope heating stage (temperatureprogrammed), with a programmed heating rate of approximately $2{ }^{\circ} \mathrm{C} \mathrm{min}{ }^{-1}$ from room temerature up to $300^{\circ} \mathrm{C}$. Cloud point was registered as the initiation temperature at which separated domains were visible using the maximum magnification of the optical microscope.

Infrared Spectroscopy. Fourier transform infrared spectroscopy (FT-IR, Nicolet Magna-560) was used for investigating possible molecular interactions between the constituents. Spectra were obtained at $2 \mathrm{~cm}^{-1}$ resolution and averages of spectra were obtained from at least 64 to 200 scans (for enhanced signal) in the standard wavenumber range of $400-4000 \mathrm{~cm}^{-1}$. All the FT-IR samples were cast as thin films of proper thin and uniform thickness directly on $\mathrm{KBr}$ pellets kept at $45^{\circ} \mathrm{C}$. Subsequently, the IR measurements were performed on the cast samples at ambient temperature.

Scanning Electron Microscopy. Morphology (fracture surface) of blends was examined using a scanning electron microscope (SEM, Model JEOL JXA-840). The blend film samples for scanning electron microscopy were solution-cast to be thick enough so that fracture surface of the thickness (cross section) could be conveniently examined. The fractured blend samples were coated with gold by vapor deposition using a vacuum sputterer.

\section{RESULTS AND DISCUSSION}

\section{Thermal Behavior and Phase Structure}

Casting of PCL/PBzMA polymer solution with THF at any temperatures at or above ambient was found to yield transparent, uniform films. When observed using an optical microscope, the blend films of all compositions by THF casting (at either 25 or $45^{\circ} \mathrm{C}$ ) were completely free of any haziness or heterogeneous domains. DSC analysis was performed on the THF-cast $\left(45^{\circ} \mathrm{C}\right)$ samples to reveal their glass transition behavior. It was expected that the thermal behavior of blends samples THF-cast at $25^{\circ} \mathrm{C}$ would be similar; thus DSC characterization was performed only on the $45^{\circ} \mathrm{C}$ THF-cast samples. For a uniform thermal history, all thermograms are the results of second runs after quenching from temperatures just above $T_{\mathrm{g}}$.

Figure 1 shows DSC thermograms revealing one apparent $T_{\mathrm{g}}$ (albeit somewhat broadened for some intermediate compositions) for PCL/PBzMA blends of a wide range of compositions, as indicated in the curves. The $T_{\mathrm{g}}$ (on-set) of blends initially increases only slightly, but then more significantly with increasing PBzMA content in PCL/PBzMA blend. As the $T_{\mathrm{g}}$ 's of neat polymers are located well apart $\left(-70\right.$ and $56^{\circ} \mathrm{C}$, respectively), $T_{\mathrm{g}}$ resolution was not a problem. All the thermograms clearly show that there is only one $T_{\mathrm{g}}$ for each composition, and that the only $T_{\mathrm{g}}$ is compositiondependent. By applying the conventional $T_{\mathrm{g}}$ criterion for determining phase miscibility, the blend is apparently miscible. However, the $T_{\mathrm{g}}$ broadening suggests that the 


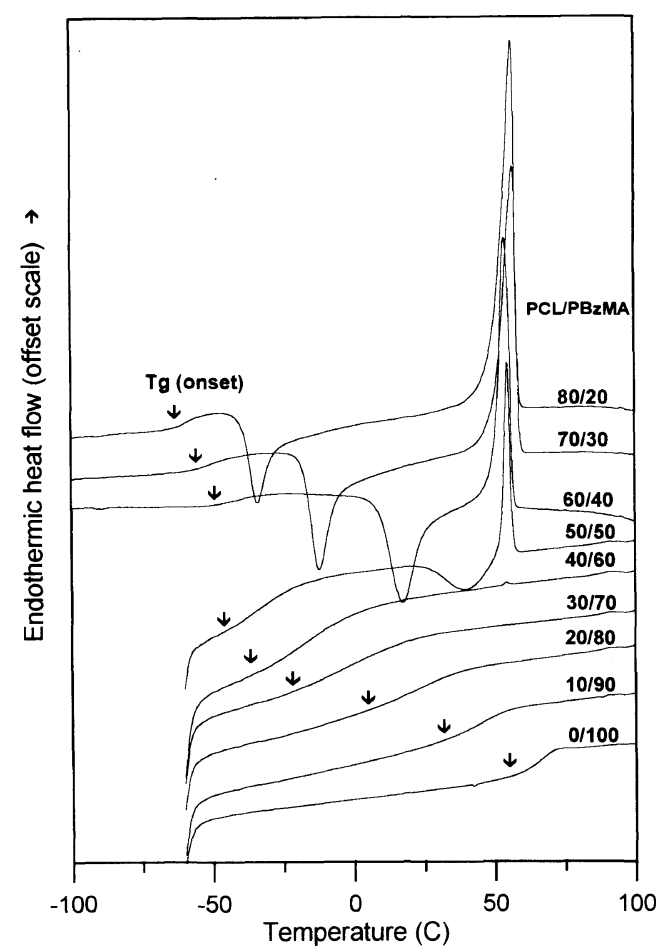

Figure 1. DSC thermograms revealing one apparent $T_{\mathrm{g}}$ for PCL/ PBzMA blends of nine different compositions, as indicated in the curves. The arrow marks where $T_{\mathrm{g}}$ is.

Table I. Glass transition temperature and transition breadth of PCL/PBzMA blends

\begin{tabular}{cll}
\hline \% PBzMA & \multicolumn{1}{c}{$T_{\mathrm{g}} /{ }^{\circ} \mathrm{C}$} & Transition breadth $/{ }^{\circ} \mathrm{C}$ \\
\hline 0 & $-65--70$ (literature) & - (highly crystalline) \\
40 & -51 & 26 \\
50 & -45.2 & 30 \\
60 & -39.0 & 33 \\
70 & -24.3 & 38 \\
80 & 4.4 & 33 \\
90 & 30.4 & 23 \\
100 & 56 & 13 \\
\hline
\end{tabular}

scale of mixing might have a limit, and that various scales of molecular aggregation might exist. The term "aggregation" here means segmental neighboring of the same molecules (or segments) in larger domains or greater probability than those of the different molecules.

Table I lists the composition dependence of transition breadth of $T_{\mathrm{g}}$. The broadening of $T_{\mathrm{g}}$ for blend samples of intermediate compositions suggests that some extent of homo-molecular aggregation might exist. The extent of intermolecular interactions might be only barely sufficient to ensure a homogeneous polymer mixtures of a limited scale of mixing $v s$. various extents of homomolecular aggregation depending on compositions. Results of investigation on molecular interactions using FT-IR will be discussed in latter sections.

To examine quantitative trend of $T_{\mathrm{g}}$ variation with composition, the onset $T_{\mathrm{g}}$ of each blend was plotted. The $T_{\mathrm{g}}$ data of the whole composition range exhibited a normally expected negative deviation from the linearity relationship. Thus, the Gordon-Taylor equation (or Kelley-Bueche model if volume fraction is used) was used for fitting with data ${ }^{13}$ :

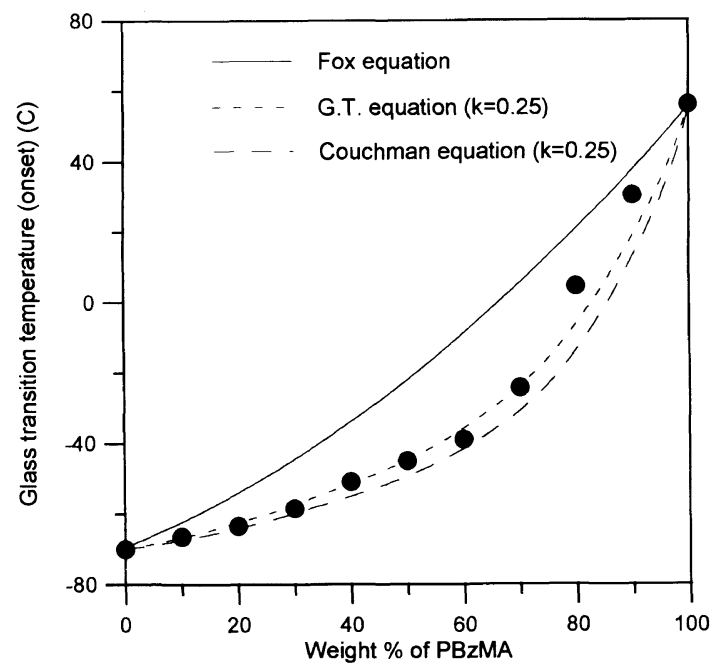

Figure 2. G-T or Couchman model fitting with the $T_{\mathrm{g}}$ data of PCL/PBzMA in the whole composition range.

$$
T_{\mathrm{g}}=\left(\omega_{1} T_{\mathrm{g} 1}+k \omega_{2} T_{\mathrm{g} 2}\right) /\left(\omega_{1}+k \omega_{2}\right)
$$

where $\omega_{i}$ is the mass (weight) fraction of polymer component $\mathrm{i}$, and $k=\Delta C_{\mathrm{p} 2} / \Delta C_{\mathrm{p} 1}$, i.e., ratio of heat capacity change of PCL polymer (low- $T_{\mathrm{g}}$ component, $T_{\mathrm{g} 1}=-70^{\circ} \mathrm{C}$ ) to the change of PBzMA (high- $T_{\mathrm{g}}$ component, $T_{\mathrm{g} 2}=56^{\circ} \mathrm{C}$ ) at $T_{\mathrm{g}}$. Note that $k=1.0$ in the Gordon-Taylor model leads to a limiting case of linear relationship of $T_{\mathrm{g}}$ vs. composition (i.e., $T_{\mathrm{g}}=\omega_{1} T_{\mathrm{g} 1}+$ $\omega_{2} T_{\mathrm{g} 2}$ ), indicating that the systems approach a limit of excellent or perfect intermolecular interactions. Another model, the Couchman equation, is also commonly used to describe the $T_{\mathrm{g}}$ behavior or polymer mixtures ${ }^{14}$ :

$$
\ln T_{\mathrm{g}}=\left[\omega_{1} \ln \left(T_{\mathrm{g} 1}\right)+k \omega_{2} \ln \left(T_{\mathrm{g} 2}\right)\right] /\left(\omega_{1}+k \omega_{2}\right)
$$

in which all symbols have the same meaning as those in G-T model. The parameter $(k)$ in either the Gordon-Taylor or Couchman equation has been suggested to correlate with the intensity of interactions between the constituent molecular segments. ${ }^{15}$

Figure 2 shows fitting of the G-T or Couchman model to the $T_{\mathrm{g}}$ data of PCL/PBzMA in the whole composition range. Although the negative deviation from linearity is not completely symmetrical, a reasonable fitting with the G-T equation could be obtained and the best fitted parametric value was found to be about $k=\sim 0.25$ by fitting with the entire range of the $T_{\mathrm{g}}$-composition data. Similar behavior of asymmetry in the $T_{\mathrm{g}}$-composition relationship has also been commonly reported in the literature for several other miscible blend systems comprising a semicrystalline polymer as one constituent, e.g., the blend of poly(butylene terephthalate) (PBT) with poly(ether imide) (PEI). ${ }^{16}$ Regardless the noted asymmetry, similarly, an equally reasonable fitting with the Couchman equation was obtained with the same value of $k=0.25$, a value almost same as the fitting obtained from the $\mathrm{G}-\mathrm{T}$ model. The relatively low value of $k$ suggests that the molecular interactions are not particularly strong or specific. This may be quite expected as the intermolecular interactions between the carbonyl groups of different polymers would not be any stronger than those among homopolymers.

For the blends of low PBzMA contents (i.e., PCL-rich, 

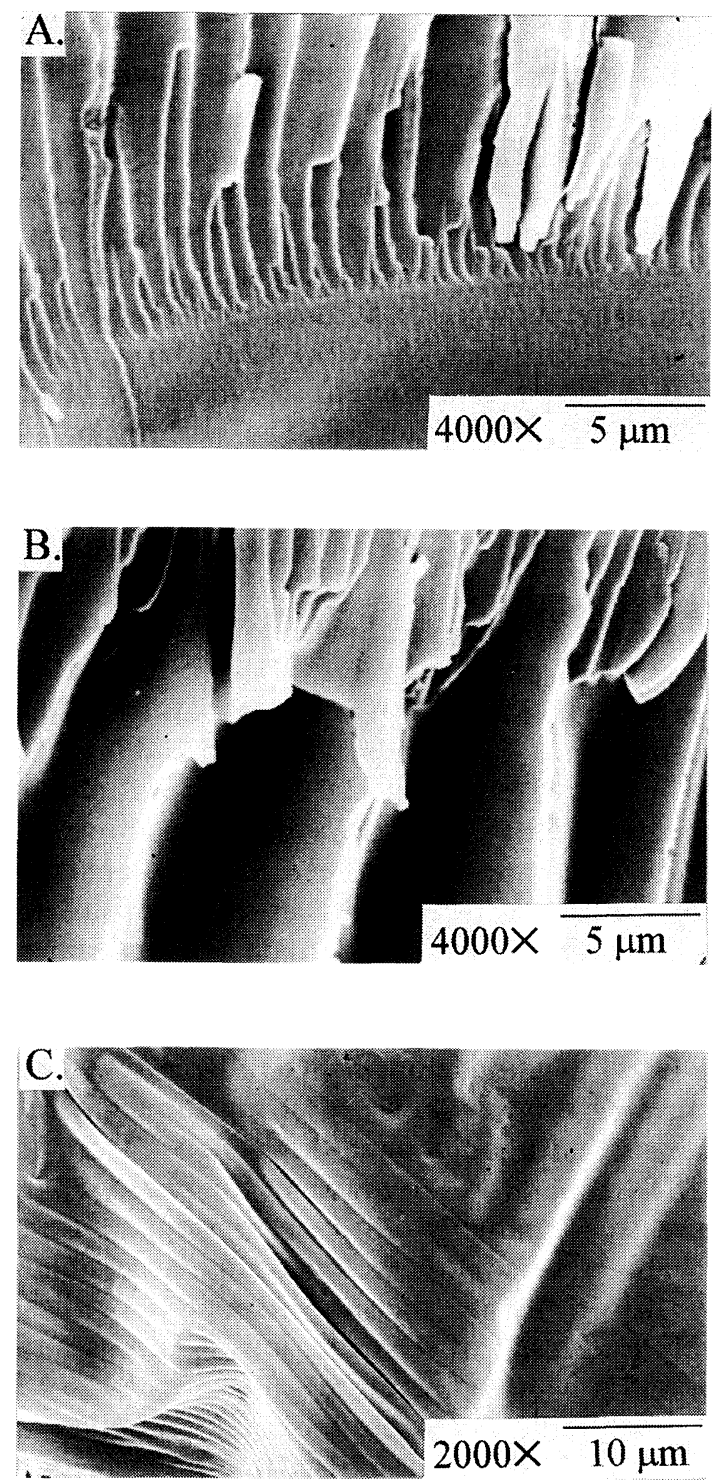

Figure 3. SEM micrographs of PCL/PBzMA of three representative compositions: (A) 10/90, (B) 20/800, and (C) 50/50.

e.g., $\mathrm{wt} \%>70$ ), there exists a leveling trend that an increase in the PBzMA content leads to sluggish increase in blend's $T_{\mathrm{g}}$. However, outside this range, the blend $T_{\mathrm{g}}$ is sensitive to composition; that is, for blends of higher PBzMA contents, the $T_{\mathrm{g}}$-composition relationship largely follows the prediction of the Gordon-Taylor equation. If the cold crystallization temperature $\left(T_{\mathrm{c}, \mathrm{c}}\right)$ is taken as an indication of easeness of chain mobility at above $T_{\mathrm{g}}$, changes in blend's $T_{\mathrm{c}, \mathrm{c}}$ reflect effects of neighboring segments. The peak location of cold-crystallization $\left(T_{\mathrm{c}, \mathrm{c}}\right)$ is seen to rise considerably with increasing PBzMA content. That is, within the range of leveling trend of blend $T_{\mathrm{g}}$ in the PCL-rich composition range, the composition dependence of cold crystallization peak (of PCL component) is quite significant with increasing PBzMA content in the blend. The result of changes in the cold crystallization temperature suggested that there existed intimate molecular mixing (in amorphous regions) between the PCL and PBzMA polymer chain segments, which causes chain stiffening of PCL molecular segments and result in elevation of coldcrystallization temperatures.

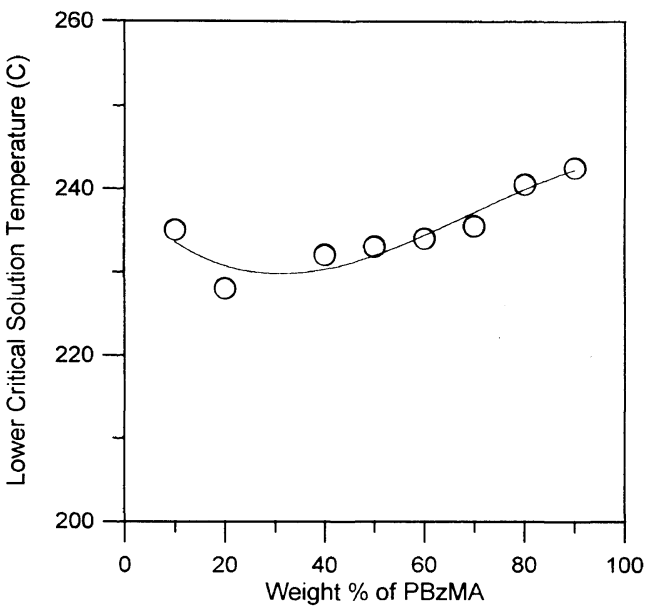

Figure 4. Cloud point (initiation) of PCL/PBzMA blends as a function of composition.

Furthermore, fracture surface morphology was directly observed using SEM. Figure 3 shows the SEM micrographs of the PCL/PBzMA blend samples of three compositions: (A) 10/90, (B) 20/800, and (C) 50/50 (weight ratios), respectively. Other compositions were also characterized; for brevity, only three samples are illustrated. SEM characterization was performed on fully amorphous, PBzMA-rich, blend compositions as PCL crystallization in PCL-rich compositions occurred at ambient temperature and presence of crystallinity complicated the observation of morphology. The three graphs clearly show no discernible heterogeneity in these solvent-cast blend samples. Thus, the SEM result provides an additional supportive evidence on phase homogeneity in this blend system.

\section{Phase Separation as Elevated Temperatures}

The THF-cast PCL/PBzMA blend samples were visually clear and free of any heterogeneous domains when examined using the optical microscope. For some miscible polymer-polymer blends with low levels of interactions, cloud point transition has been common. To monitor whether or not there existed a cloud-point transition in this polymer mixture system, changes of optical clarity of the samples of various compositions with respect to temperature were inspected at step-wise elevating temperatures up to where a cloud transition was observed or where degradation occurred $\left(300^{\circ} \mathrm{C}\right.$ or up). The onset temperature of cloudiness in the originally transparent samples upon slow heating was recorded as the cloud point.

Figure 4 shows the curve of cloud point (initiation) of PCL/PBzMA blend as a function of composition. The shape of the cloud point curve is gently convex up at two ends with a slightly asymmetric shape, with the cloud point transition for the $90 / 10$ and $10 / 90$ compositions occurring at about $235-240^{\circ} \mathrm{C}$ and the $80 / 20$ composition showing a minimum cloud point of about $230^{\circ} \mathrm{C}$. As the phase structure of the blend at below the cloud point was determined to be completely homogeneous and free of any micro heterogeneity, the occurrence of a cloud point phenomenon suggests a thermodynamic phase separation from original miscibility. That is, this cloud point phenomenon is attributed to its thermo- 

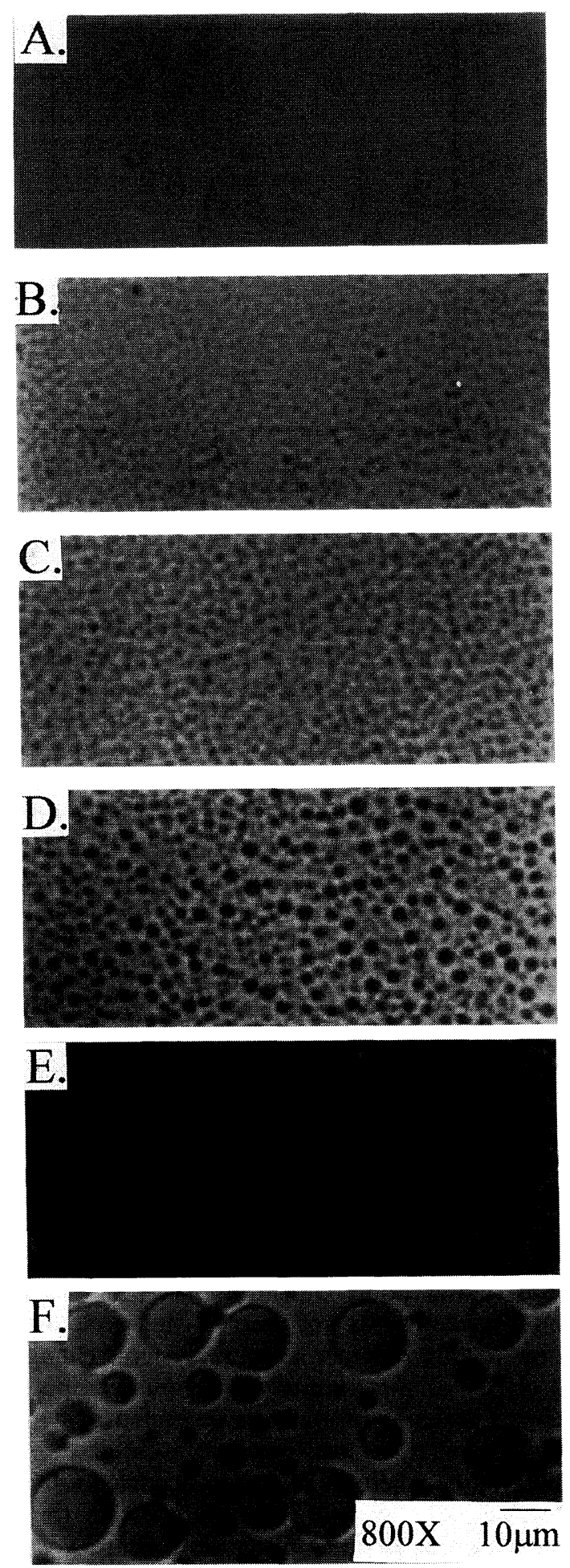

Figure 5. Observed phase domains of PCL/PBzMA (50/50) at $240^{\circ} \mathrm{C}$ for various times: (a) 0 (unheated), (b) $40 \mathrm{~s}$, (c) $2 \mathrm{~min}$, (d) $8 \mathrm{~min}$, (e) $15 \mathrm{~min}$, and (f) $25 \mathrm{~min}$.

dynamic behavior of LCST.

Development of phase domains was monitored by examining the initiation and growth of the phase domains of the PCL/PBzMA blend sample using the optical microscopy. Photos were taken after the samples were heated to $240^{\circ} \mathrm{C}$ for various time from 0 to $30 \mathrm{~min}$. Figure $5(\mathrm{a}-\mathrm{f})$ shows the optical micrographs $(800 \times)$ revealing the size and distribution of the phase domains of the PCL/PBzMA (50/50) at above LCST for various times: (a) 0 (unheated), (b) $40 \mathrm{~s}$, and (c) $2 \mathrm{~min}$, (d) $8 \mathrm{~min}$, (e) $15 \mathrm{~min}$, and (f) $25 \mathrm{~min}$. Diagram-A shows the unheated (as-cast) sample appearing homogeneous and free of phase separation. Once heated to $240^{\circ} \mathrm{C}$, phase separation was initiated in the originally homogeneous polymer mixture and the size of phase domains steadily increased with time of heating at $240^{\circ} \mathrm{C}$. At the beginning of phase separation, the size was about 1 micron or less (Diagram-B), showing an unconnected spheroidal domains from early time $(0-40 \mathrm{~s})$. With the heating time extended to $25 \mathrm{~min}$, the domain size increased to about $10 \mu \mathrm{m}$ or larger (Diagram-F). The pattern of phase domain remains spherical throughout the heating time, which indicates a nucleation and growth (NG) mechanism (unconnected spheres) in the boundary of binodal curve.

Figure 6 shows the optical micrographs $(800 \times)$ of the development of phase domains of the PCL/PBzMA of another composition (30/70) at above LCST $\left(240^{\circ} \mathrm{C}\right)$ for various times: (a) 0 (unheated), (b) $40 \mathrm{~s}$, and (c) $2.5 \mathrm{~min}$, (d) $8 \mathrm{~min}$, (e) $15 \mathrm{~min}$, and (f) $25 \mathrm{~min}$. The phase development of the PCL/PBzMA blend at above LCST for the $30 / 70$ composition is seen to be comparable to that for the 50/50 composition in the previous figure. Again, the pattern of phase domain remains spherical, which indicates phase separation via a nucleation \& growth (NG) mechanism upon heating to above LCST.

The LCST phenomenon in PCL/PBzMA was found to be reversible upon slowly lowering the temperature. This means that upon cooling down slowly from above the LCST temperatures to ambient, the phase-separated structure was gradually restored to original transparency. This reversibility is kinetically accessible and readily driven to its thermodynamics-favored state, probably as a result of relatively low $T_{\mathrm{g}}$ in this mixture system and a wide temperature gap between the LCST and $T_{\mathrm{g}}$. With the favorable molecular mobility attributed to low $T_{\mathrm{g}}$, restoration back to homogeneity from phase separation at above LCST became easily accessed. Furthermore, the restoration of phase homogeneity upon cooling down from LCST indicates that no chemical reactions were involved in the phase separation at above LCST. No trans esterifications between PCL and PBzMA, for examples, were observed at $240^{\circ} \mathrm{C}$.

Evidence of phase reversibility was examined by characterizing the $T_{\mathrm{g}}$ behavior of the blend samples that were subjected to heating to $\operatorname{LCST}\left(240^{\circ} \mathrm{C}\right.$ for $\left.5 \mathrm{~min}\right)$ and cooling back to ambient. Figure 7 shows comparison of $T_{\mathrm{g}}$ curves of two PCL/PBzMA compositions: (a) 20/80 as-cast, (b) $20 / 80$, heated $240^{\circ} \mathrm{C}$ for $5 \mathrm{~min}$ and cooled, and (c) $30 / 70$ as cast, (d) $30 / 70$, heated $240^{\circ} \mathrm{C}$ for $5 \mathrm{~min}$ and cooled. Apparently, the $T_{\mathrm{g}}$ for the as-cast sample is almost the same as the sample (of same composition) subjected to LCST and then cooled back to ambient. A single $T_{\mathrm{g}}$, at the same temperature with similar breadth, is observed in the as-cast sample as well as the same sample subjected to LCST for $5 \mathrm{~min}$ and then cooled. The thermal transition evidence indicates that restoration of phase homogeneity is kinetically accessible for the polymer mixtures being cooled from LCST.

Additionally, if quenched at extremely high rates from above LCST to below $T_{\mathrm{g}}$, the blend stayed temporarily at a state of phase separation. However, if the quenched 

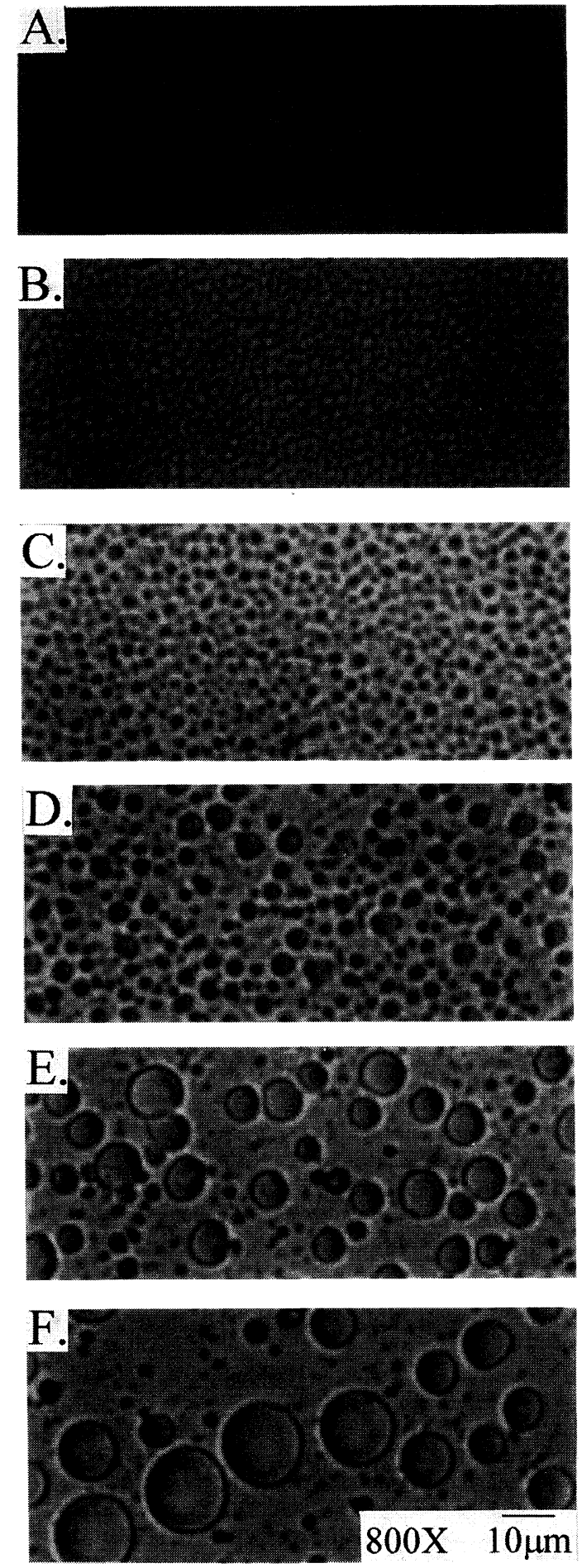

Figure 6. Observed phase domains of PCL/PBzMA (30/70) at above $240^{\circ} \mathrm{C}$ for various times: (a) 0 (unheated), (b) $40 \mathrm{~s}$, (c) $2.5 \mathrm{~min}$, (d) $8 \mathrm{~min}$, (e) $15 \mathrm{~min}$, and (f) $25 \mathrm{~min}$.

blend sample was subsequently annealed for sufficient times at a proper temperature above $T_{\mathrm{g}}$, the phase separation state could be reversed to its thermodynamic state of phase miscibility. DSC characterization on such samples revealed a single $T_{\mathrm{g}}$, and optical microscopy cofirmed reversibility to homogeneity in the annealed blend samples. For brevity, the DSC traces or optical micrographs are not shown.

\section{Molecular Interactions vs. Aggregation}

Figure 8 shows the IR spectra of the carbonyl $(\mathrm{C}=\mathrm{O})$

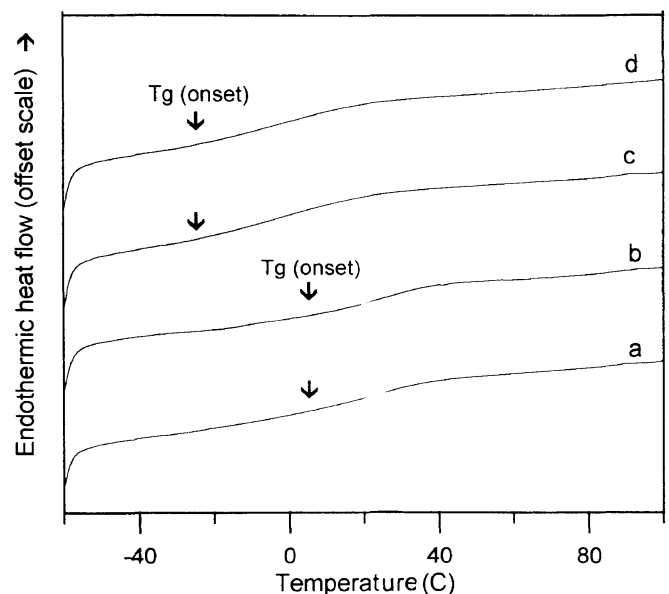

Figure 7. Thermal evidence showing reversibility of phase separation to miscible state for PCL/PBzMA of 20/80 and 30/70 compositions: (a) \& (c) as-cast samples (b) \& (d) samples heated to $240^{\circ} \mathrm{C}$ for $5 \mathrm{~min}$ then cooled by quenching.

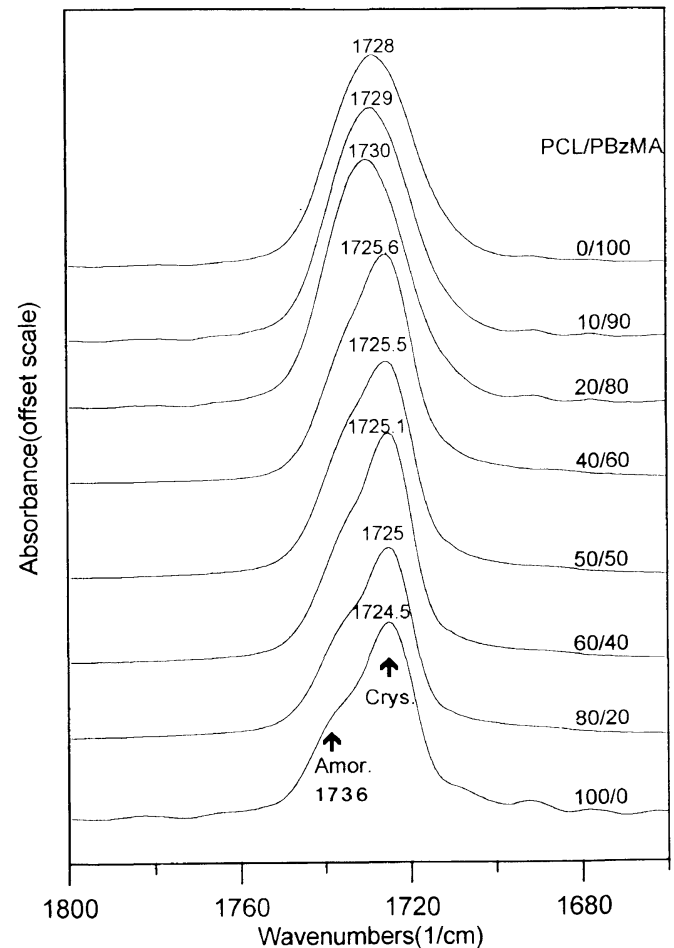

Figure 8. IR spectra of the carbonyl $(\mathrm{C}=\mathrm{O})$ absorbance peak for the PCL/PBzMA samples (bottom to top): (a) neat PCL, (b) 80/20, (c) 60/40, (d) 50/50, (e) 40/60, (f) 20/80, (g) 10/90, and (h) neat PBzMA.

absorbance peak for the PCL/PBzMA samples (bottom to top): (a) neat PCL, and (b) 80/20, (c) 60/40, and (d) 50/50, (e) 40/60, (f) 20/80, (g) 10/90, and (h) neat PBzMA. For the neat PCL sample (Spectrum-a), a prominent doublet absorption peak is seen to be located at 1736$1725 \mathrm{~cm}^{-1}$. The crystalline $\mathrm{C}=\mathrm{O}$ absorption peak of PCL is known to be located at approximately 1724.5 $\mathrm{cm}^{-1}$. Thus this crystalline absorbance peak remains at the same wavenumber for all blend compositions and for the neat PCL as the crystalline lamellar chains are not mixed with PBzMA. Next to the crystalline absorption, a shoulder peak is observed at $1736 \mathrm{~cm}^{-1}$, which is attributed to the carbonyl group of the amorphous portion of PCL. The carbonyl group of neat 
PBzMA is seen to be located at $1728 \mathrm{~cm}^{-1}$. The carbonyl group of the blend of various compositions exhibits a gradual shifting between $1728 \mathrm{~cm}^{-1}(\mathrm{C}=\mathrm{O}$ of PBzMA) and $1736 \mathrm{~cm}^{-1}$ (amorphous $\mathrm{C}=\mathrm{O}$ of PCL) as the composition is changed. The gradual peak shifting suggests that molecular chains in the amorphous portions are mixed. Note that the shoulder peak (the carbonyl group of the crystalline portion of PCL) no longer exists in the PCL/PBzMA blend samples of 10/90 and 20/80 as these PBzMA-rich blend compositions are fully amorphous with no crystalline PCL domain. The overall shifting trend of $\mathrm{C}=\mathrm{O}$ peak in the amorphous region and disappearance of the crystalline $\mathrm{C}=\mathrm{O}$ peak for blends of higher PBzMA contents are similar to those observed in the PVC/PCL systems reported earlier by Coleman and Zarian. ${ }^{17}$

\section{CONCLUSION}

PCL and PBzMA are two different polymers containing an ester group at different positions, i.e., one in the main chain and the other in the pendant group, respectively, which have been found to exhibit miscibility to each other in the full composition range. The binary polymer mixture forms a miscible blend with a homogeneous phase structure. It is concluded that the blend phase behavior is miscibility with non-specific intermolecular interactions. The rare miscibility in the carbonyl-containing polymer pairs may be a result of closely-matched polarity of these two carbonyl-containing polymers. The thermal transition behavior proved a single $T_{\mathrm{g}}$ that is composition-dependent although various extents of $T_{\mathrm{g}}$ broadening were also observed. The segmental scales are not particularly fine and some minor homo-molecular aggregation might be possible. The FT-IR results did not show signiffcant interactions between functional groups. The lack of significant peak shifting $(\mathrm{C}=\mathrm{O}$ or other functional groups) may be associated with a fact that a low percentage of PBzMA segments engaging in intermolecular interactions, which is a phenomenon similar to what was reported for a miscible poly(styrene-co-acrylonitrile)/poly(methyl methacrylate) blend. ${ }^{18}$ Although Coleman, et al. ${ }^{19}$ proposed a concept of non-hydrogen-bonded solubility parameter for prediction of polymer-polymer miscibility and observed that two polymers with close values of non-hydrogenbonded solubility parameters are likely miscible, it is interesting to note that the literature contains very few reports of miscibility of two different polymers with carbonyl groups. In this study, PCL and PBzMA have been shown to be miscible using the conventional criteria, with probably various scales of homo-molecular aggregation in competition with hetero-molecular mixing.
Furthermore, like many miscible polymer mixtures with low interactions, phase separation (cloud point) took place in the PCL/PBzMA system upon heating to about $230-240^{\circ} \mathrm{C}$ and above, exhibiting a lower critical solution temperature. This LCST phenomenon was determined to be thermodynamically driven, and phase separation assumed an NG mechanism. Opposite to kinetic irreversibility of LCST commonly observed in most blend systems with high $T_{\mathrm{g}}$, phase separation of the present PCL/PBzMA system was kinetically reversible upon cooling slowly back to ambient in normally accessible time scales. This kinetic reversibility to thermodynamically-favored states provides evidence that the reported miscibility in PCL/PBzMA was not a result of temporary kinetic trapping during solvent evaporation. The reversibility also suggests that no chemical changes/reactions were involved in the phase separation process at above LCST.

Acknowledgment. Research grant (\#NSC 87-2216E006-005) for this study was provided by National Science Council of Executive Yun of Taiwan Government. T. K. Mandal is a post-doc fellow also sponsored by NSC of Taiwan.

\section{REFERENCES AND NOTES}

1. J. V. Koleske and R. D. Lundberg, J. Polym. Sci., Part-A-2, 7, 795 (1969).

2. L. P. McMaster, Adv. Chem. Ser., 142, 43 (1975).

3. G. L. Brode and J. V. Koleske, J. Macromol. Sci. Chem., 6, 1109 (1972).

4. D. R. Paul, J. W. Barlow, C. A. Cruz, R. N. Mohn, T. R. Wassar, D. C. Wahrmund, Am. Chem. Soc., Div. Org. Coat. Plast. Chem., Pap., 37, 130 (1977).

5. M. K. Neo and S. H. Goh, Eur. Polym. J., 27, 927 (1991).

6. D. Ma, X. Lu, R. Zhang, and T. Nishi, Polymer, 37, 1575 (1996).

7. A. Siciliano, A. Seves, T. De Marco, S. Cimmino, E. Martuscelli, and C. Silvestre, Macromolecules, 28, 8065 (1995).

8. J. S. Chiou, J. W. Barlow, and D. R. Paul, J. Polym. Sci., Part $B, 25,1459$ (1987).

9. J. M. Saldanha and T. Kyu, Macromolecules, 20, 2840 (1987).

10. M. Nishimoto, H. Keskkula, and D. R. Paul, Polymer, 32, 272 (1991).

11. M. Motowoka, H. Jinnai, T. Hashimoto, Y. Qiu, and C. C. Han, J. Chem. Phys., 99, 2095 (1993)

12. E. M. Woo and C. C. Su, Polymer, 37, 4111 (1996).

13. M. Gordon and J. S. Taylor, J. Appl. Chem., 2, 493 (1952).

14. P. R. Couchman, Macromolecules, 11, 1156 (1978).

15. R. E. Prud'homme, Polym. Eng. Sci., 22, 90 (1982).

16. E. M. Woo and S. N. Yau, Macromolecules, 30, 3626 (1997)

17. M. M. Coleman and J. Zarian, J. Polym. Sci., Polym. Phys., 17, 837 (1979).

18. K. Naito, G. E. Johnson, D. L. Allara, and T. K. Kwei, Macromolecules, 11, 1260 (1978)

19. M. M. Coleman, C. J. Serman, D. E. Bhagwager, and P. C. Painter, Polymer, 31, 1187 (1990). 